\title{
On Microscopic Mechanisms Which Elongate the Tail of Cluster Size Distributions: An Example of Random Domino Automaton
}

\author{
ZBIGNIEW CZECHOWSKI ${ }^{1}$
}

\begin{abstract}
On the basis of simple cellular automaton, the microscopic mechanisms, which can be responsible for elongation of tails of cluster size distributions, were analyzed. It was shown that only the appropriate forms of rebound function can lead to inverse power tails if densities of the grid are small or moderate. For big densities, correlations between clusters become significant and lead to elongation of tails and flattening of the distribution to a straight line in $\log -\log$ scale. The microscopic mechanism, given by the rebound function, included in simple 1D RDA can be projected on the geometric mechanism, which favours larger clusters in 2D RDA.
\end{abstract}

Key words: Long tails, SOC, Cellular automata.

\section{Introduction}

Long tail distributions appear in many natural phenomena, e.g., in geophysics we can mention: size distribution of earthquakes, seismic faults, volcanic eruptions, and floods or hurricanes. We have only a limited understanding of the physical origins of long tails in these processes. Some models were introduced to explain this behaviour. In particular, SOC models, given by appropriate cellular automata, were proposed. They generate power laws; however, it is not exactly clear what mechanism is responsible for these long tail distributions in the models. However, it was frequently claimed that as a result of repetitive action of avalanches, the spatial correlations between different parts of the system appear, which lead to power distributions, like in critical phenomena. Interesting reviews of various approaches

1 Institute of Geophysics Polish Academy of Sciences, Księcia Janusza 64, 01-452 Warsaw, Poland. E-mail: zczech@igf.edu.pl corresponding to this problem are presented in (SoRNETTE 2006; AsChWANDEN 2013).

The goal of this paper is to show that microscopic rules of cellular automata should be considered as the main cause of long tail distributions. In order to prove this, we use the simple 1D cellular automaton, which is described by analytical equations. This feature enables us to make a clear analytical investigation of a role for microscopic rules in generating long tails. The automaton was introduced and analyzed in our previous papers (CZECHOWsKi and BIAŁECKI 2012a, b; BiaŁecki and CZechowski 2013; BiaŁeCKi 2013).

In Sect. 2 the rules and equations of the 1D model are briefly presented. The influence of some details of microscopic rules of the automaton and correlations on a shape of the cluster size distribution function is analyzed in Sect. 3. Section 4 introduces a similar 2D cellular automaton, in which inverse power tails have a geometric origin. In Sect. 5 we show that the simple $1 \mathrm{D}$ automaton can mimic and explain the behavior of a more complex 2D model.

\section{The Model}

The random domino automaton (RDA) is defined on a grid of $N$ cells (e.g., 1D or 2D with different geometry), in which it is determined what is the neighbourhood of the cells. Cells can be in two states: empty or occupied by a single point. The rules of RDA on the grid are as follows: at each time step a point hits a randomly chosen cell; if this cell is empty, it becomes occupied with probability $v$ or with probability $1-v$ and the point is rebounded. If the cell belongs to a cluster of $i$ neighbouring occupied cells then with probability $\mu(i)$ all cells of the cluster become empty (the avalanche of size $i$ is triggered) or 
with probability $1-\mu(i)$ the point is rebounded from the grid. After an initial saturation process, the automaton reaches a statistically stationary state. The RDA may be treated as an extension (see for details Czechowski and BiaŁecki 2012a; BiaŁecki and Czechowski 2013) of the forest fire model studied in PACZUSKI and BAK (1993).

In the case of a 1D grid, in a statistically stationary state, the following set of equations for size distribution of occupied clusters $n_{i}$ was derived (BIAteCKI and CZECHOWSKI 2013):

$$
\begin{aligned}
n_{1}= & \frac{1}{\mu(1) / v+2}\left((1-\rho) N-2 n+n_{1}^{0}\right) \\
n_{2}= & \frac{1}{2 \mu(2) / v+2}\left(1-\frac{n_{1}^{0}}{n}\right) \\
n_{i}= & \frac{1}{i \mu(i) / v+2}\left[2\left(1-\frac{n_{1}^{0}}{n}\right) n_{i-1}+n_{1}^{0} \sum_{k=1}^{i-2} \frac{n_{k} n_{i-1-k}}{n^{2}}\right] \\
& i \geq 3,
\end{aligned}
$$

where $\rho$ (density of occupied cells), $n$ (number of clusters), and $n_{1}^{0}$ (number of empty clusters of size 1) are expressed in terms of $n_{i}, \mu(i)$ and $v$ :

$$
\begin{array}{r}
\rho=\frac{1}{N} \sum_{i \geq 1} i n_{i}, \quad n=\sum_{i \geq 1} n_{i}, \\
n_{1}^{0}=\frac{2 n}{3+\frac{2}{v n} \sum_{i \geq 1} i \mu(i) n_{i}} .
\end{array}
$$

For given $N, \mu(i)$ and $v$, Eq. (1) with formulas (2) form a closed set of implicit equations for $n_{i}$.

In this paper we assume the rebound function $\mu(i)$ of the form $\mu(i)=\delta / i^{\sigma}$ with $\sigma=0,1$ and 2 . Equation (1) may present a simple recurrence if we can find $n, \rho$, and $n_{1}^{0}$. This is possible for $\sigma=1$ or $\sigma=2$, because equations for moments

$$
m_{z}=\frac{1}{N} \sum_{i \geq 1} i^{z} n_{i}, \quad m_{z}^{\prime}=\frac{1}{N} \sum_{i \geq 1} \frac{\mu(i)}{v} i^{z} n_{i}
$$

derived (Biatecki and Czechowski 2013) in the following form

$$
\begin{aligned}
m_{z+1}^{\prime}= & 1-m_{1}-2 m_{2}+2 \sum_{k=0}^{z-1}\left(\begin{array}{l}
z \\
k
\end{array}\right) m_{k} \\
& +\frac{2}{3 m_{0}+2 m_{1}^{\prime}} \sum_{l, p=1}^{l+p \leq z}\left(\begin{array}{c}
z \\
l+p
\end{array}\right)\left(\begin{array}{c}
l+p \\
l
\end{array}\right) m_{l} m_{p},
\end{aligned}
$$

can be reduced to a closed set of two equations (for $z=0$ and $z=1$; in the case $\sigma=1$ ),

$$
\begin{aligned}
& \theta m_{0}=1-m_{1}-2 m_{0} \\
& \theta m_{1}=1-m_{1}
\end{aligned}
$$

or a closed set of three equations (for $z=1, z=2$, $z=3$; in the case $\sigma=2$ ).

$$
\begin{aligned}
& \theta m_{-1}=1-m_{1}-2 m_{0} \\
& \theta m_{0}=1-m_{1} \\
& \theta m_{1}=1+3 m_{1}+2 m_{1}^{2} \frac{2}{3 m_{0}+2 \theta m_{-1}}
\end{aligned}
$$

where $\theta=\delta / v$. Let us note that $m_{0}=n / N$ and $m_{1}=\rho$.

On the other hand, in the case of $\sigma=0$ [i.e., $\mu(i)=\delta=$ const $]$ we have no closed set of moment equations, but $n$ and $n_{1}^{0}$ can be expressed by $\rho$ :

$$
\begin{gathered}
n(\rho)=\frac{N}{2}\left[1-\left(\frac{\delta}{v}+1\right) \rho\right], \\
n_{1}^{0}(\rho)=\frac{2 n^{2}(\rho)}{3 n(\rho)+2 N \frac{\delta}{v} \rho},
\end{gathered}
$$

where the first equation results from the balance for $n$ (see Eq. 2 in Bialecki and Czechowski 2013). Then, in order to find the stationary density, we put Eqs. (7) and (8) to the set (1) and solve numerically the following implicit equation (definition of density) for unknown variable $\rho$ :

$$
\rho=\frac{1}{N} \sum_{i \geq 1} i n_{i}(\rho) .
$$

\section{Influence of Microscopic Rules of the Automaton and Correlations on the Length of the Tail of Cluster Size Distribution}

We check how some details of microscopic rules of the automaton, such as a form of function $\mu(i)$, the parameter $\theta=\delta / v$, and possible correlations between clusters, can influence the shape of the cluster distribution function.

\subsection{Role of a Form of Function $\mu(i)$}

We assume $\mu(i)=\delta / i^{\sigma}$ and analyze the following three cases: 


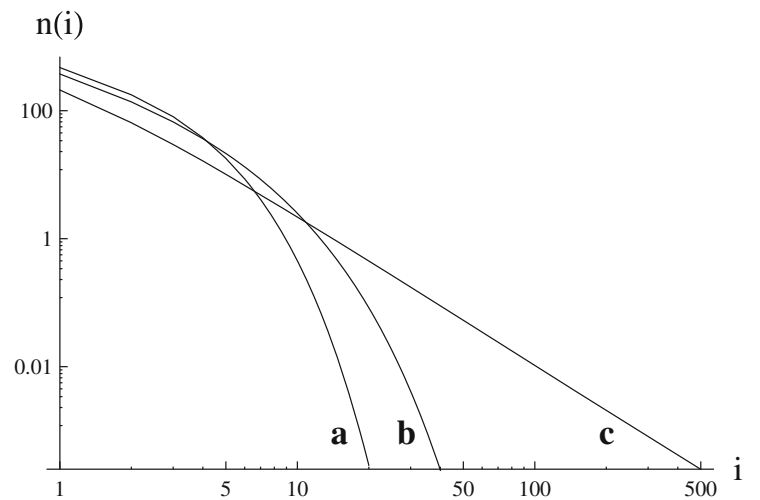

Figure 1

Cluster size distributions $n_{i}$ calculated from Eq. (1) with $\mu(i)=\delta / i^{\sigma}$ for three cases: $\mathbf{a} \sigma=0$ and $\theta=0.68, \mathbf{b} \sigma=1$ and $\theta=1.81$, and c $\sigma=2$ and $\theta=7$. For each case the stationary density is $\rho \approx 0.3559$
(a) $\sigma=0$
(b) $\sigma=1$
(c) $\sigma=2$.

Therefore, as it has been discussed in Sect. 2, the cluster size distribution $n_{i}$ can be found from the recurrent Eq. (1). For each case we set for the stationary density the same moderate value $\rho=0.3559$; this condition is realized for: $\theta=7$ when $\sigma=2, \theta=1.81$ when $\sigma=1$, and $\theta=0.68$ when $\sigma=0$. Figure 1 shows cluster size distributions $n_{i}$. The discrete distributions are presented by continuous lines for better clarity. For $\sigma=0$ (constant $\mu$ ) a short-tail exponential distribution is obtained. However, if the rebound function $\mu(i)$ is a decreasing function then a lengthening of the tail is observed-the faster the decrease the longer the tail. For $\sigma=2$ and $\theta=7$ the tail is inverse-power with the exponent about -2.323 .

Explanation of the mechanism is simple: the function $\mu(i)$ describes the probability that the particle, which hits a randomly chosen occupied cell belonging to a cluster of size $i$, triggers the avalanche (the cluster disappears). If $\mu(i)$ decreases with a cluster size then, in that trial, a probability of survival of larger clusters is greater than for smaller clusters. This mechanism leads to the bigger input of larger clusters in the distribution, i.e., to longer tails.
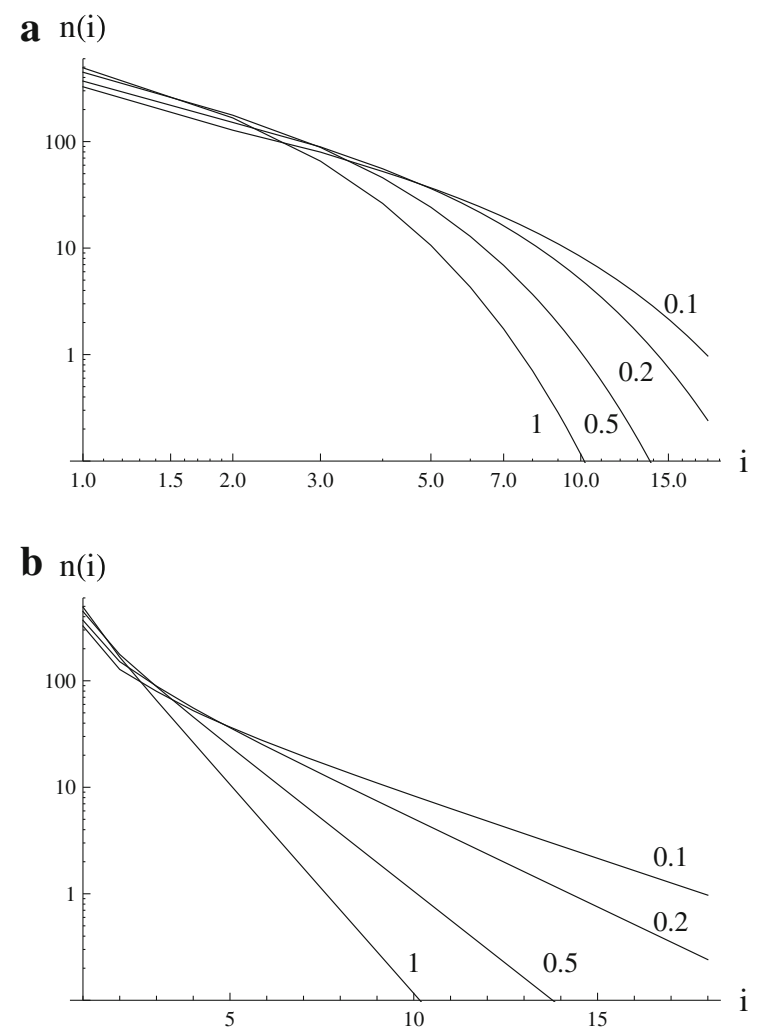

Figure 2

a Cluster size distributions $n_{i}$ calculated from Eq. (1) with $\mu(i)=\delta$ for four assumed values of parameter $\theta=1,0.5,0.2$ and 0.1 (in $\log -\log$ scale). b Cluster size distributions $n_{i}$ calculated from Eq. (1) with $\mu(i)=\delta$ for four assumed values of parameter $\theta=1$, $0.5,0.2$ and 0.1 (in lin-log scale to show exponential tails)

\subsection{Role of Parameter $\theta=\delta / v$}

When the form of function $\mu(i)=\delta / i^{\sigma}$ and the exponent $\sigma$ is established, then the value of parameter $\delta$ itself can have an influence on the shape of the cluster size distribution. In all formulas the parameter is appearing in the ratio $\theta=\delta / v$; therefore, we investigate an influence of changing $\theta$ separately for the three cases $(\mathrm{a}-\mathrm{c})$.

In case (a) decreasing parameter $\theta$ does not change an exponential dependence of the tail of cluster size distribution on the cluster size, but the exponential decrease is less and less steep (see Fig. 2a, b).

In case (b) a similar lengthening of tails is also observed (see Fig. 3a). Moreover, the widening central part of the distribution is achieving (in the 

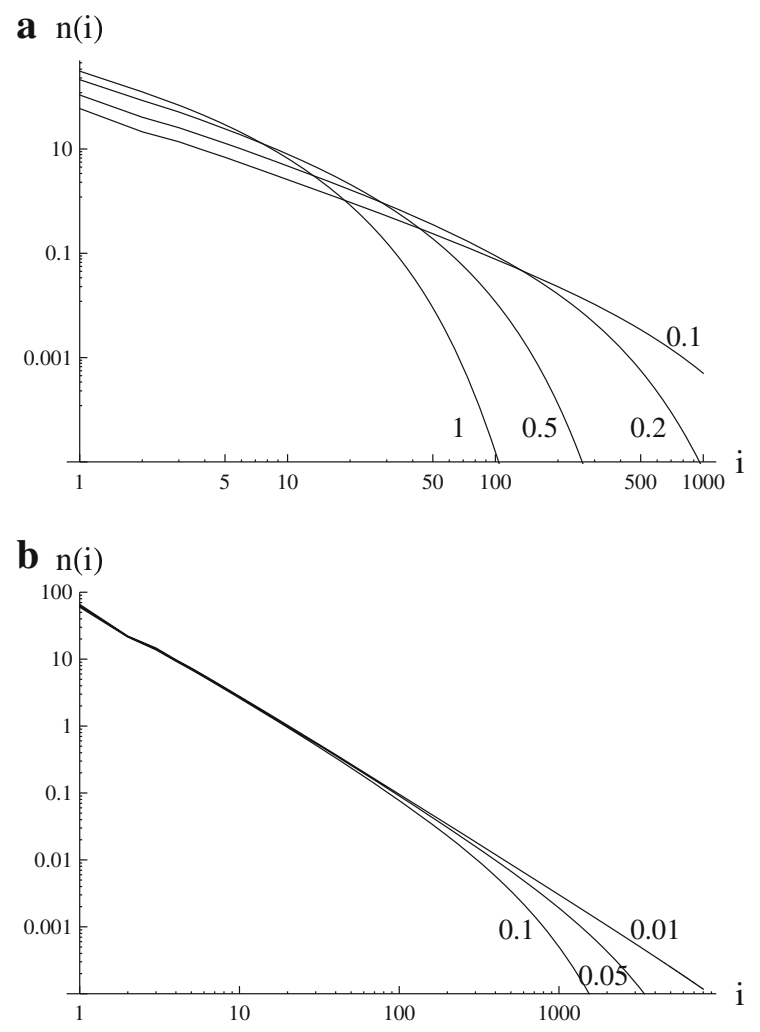

Figure 3

a Cluster size distributions $n_{i}$ calculated from Eq. (1) with $\mu(i)=$ $\delta / i$ for four assumed values of parameter $\theta=1,0.5,0.2$ and 0.1 . b Cluster size distributions $n_{i}$ calculated from Eq. (1) with $\mu(i)=\delta / i$ for three assumed values of parameter $\theta=0.1,0.05$ and 0.01 . Here the fixed product $\theta N$ is maintained when $\theta$ decreases and $N$ increases. For the case $\theta=0.01$, the power exponent is $\alpha \approx-1.5$

limit $\theta \rightarrow 0$ ) the inverse-power slope $i^{-3 / 2}$. Figure $3 \mathrm{~b}$, in which the fixed product $\theta N$ is maintained when $\theta$ decreases, clearly shows the behavior. This is in agreement with the asymptotic result of Motzkin numbers recurrence (BIAEECKI 2012a) applied to RDA.

In case (c) decreasing parameter $\theta$ causes decreasing inverse-power exponents of the tail, e.g., if $\theta$ drops from 8.0 to 7.0, the exponent drops from 2.83283 to 2.32307 (see Fig. 4). Therefore, in this case we also observe lengthening of tails with decreasing $\theta$. In Fig. 4 two graphs of $n_{i}$ are presented for each $\theta$, because the closed set (6) of three moment equations has two solutions: $\left(m_{1}^{(1)}, m_{2}^{(1)}, m_{3}^{(1)}\right)$ and $\left(m_{1}^{(2)}, m_{2}^{(2)}, m_{3}^{(2)}\right)$ if $\theta>6.936236$.

In order to explain the common (in the three cases under investigation) influence of the parameter $\theta$ on

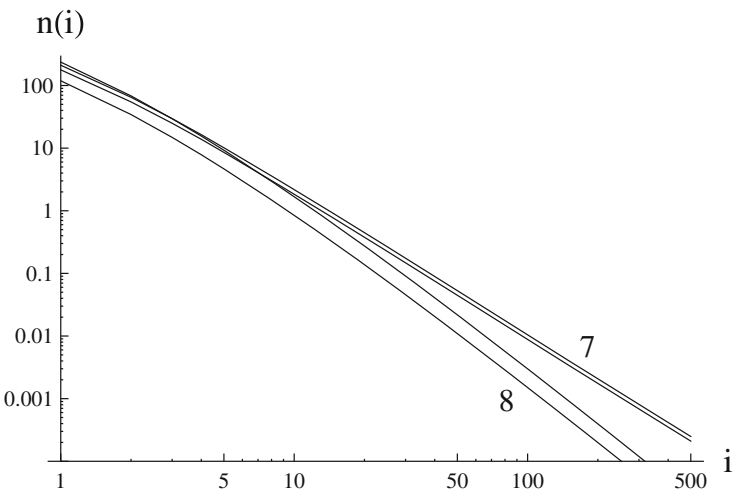

Figure 4

Cluster size distributions $n_{i}$ calculated from Eq. (1) with $\mu(i)=\delta / i^{2}$ for two assumed values of parameter $\theta=8$ and 7 . For each $\theta$ two lines are presented because Eq. (6) has two solutions. Power exponent for the pair $\theta=8$ is $\alpha \approx-2.83$ and for the pair $\theta=7$ is $\alpha \approx-2.32$

tails of cluster size distributions, we should note that decreasing $\theta$ causes increasing stationary density $\rho$ (when $\theta \rightarrow 0$ then $\rho \rightarrow 1$ ). The increase of density means reducing the number of empty cells on the grid and, as a result, it reduces a possibility of creation of clusters of size 1 (and their next growth to small clusters of size $2,3, \ldots)$. On the other hand, the probability of growth of clusters, due to hitting an empty cell on a cluster perimeter or due to joining two neighbor clusters if the particle hits the empty cell between them, increases. These mechanisms lead to the growth of the number of greater clusters at the expense of the number of small clusters (because the source of $n_{1}$ is being depleted). This is observed as the lengthening of tails.

\subsection{Role of Correlations Between Clusters}

Equation (1) was derived under the assumption that clusters are distributed independently on the grid (i.e., the length of the next cluster on the grid does not depend on the length of the previous one). The assumption can be justified only for small and moderate densities. However, for big densities the correlations can be significant. Here, we analyze the influence of correlation on a shape of the cluster size distribution function. For the test we use 1D RDA with constant $\mu(i)=\delta$, and we compare analytically computed $n_{i}$ with the results of simulations. When we 
a $\mathrm{n}(\mathrm{i})$

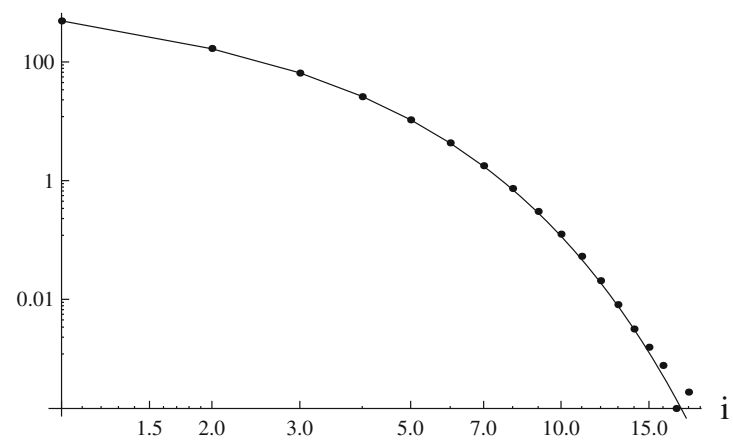

b $n(i)$

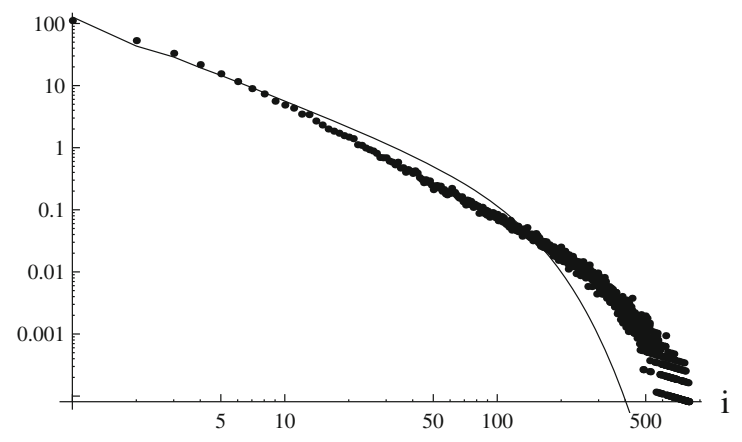

Figure 5

a Comparison of cluster size distributions $n_{i}$ calculated from Eq. (1) (line) with generated by simulation of RDA (points) for the case $\mu(i)=\delta$ and $\theta=1$. The stationary density is $\rho \approx 0.3076$. The influence of correlations is negligible. b Comparison of cluster size distributions $n_{i}$ calculated from Eqs. (1) (line) with generated by simulation of RDA (points) for the case $\mu(i)=\delta$ and $\theta=0.002$. The stationary density is $\rho \approx 0.8099$. Influence of correlations is significant. There is a lengthening of the tail, and a remarkable flattening of the simulated distribution to a straight line $(\alpha \approx$ -1.78 ) in the central part of the graph is visible

assume $\theta=1$, the moderate value of stationary density $\rho=0.3076$ is obtained. Figure 5a shows a good agreement between the analytical curve and the numerical result represented by points. This means that correlations are too weak to deform the cluster size distribution. However, for a very small $\delta=0.002$, the stationary density increases to $\rho=0.8099$ and the influence of correlations is significant. First of all, on Fig. 5b we observe a lengthening of the tail of a simulated distribution when $i>200$, but, what is more important, a remarkable flattening of the distribution to a straight line in the range $i \in(4,300)$ is evident. In log-log scale this means power-law behaviour. The exponent is about -1.78 .

Spatial correlations between different parts of the system were often taken into account as a main factor leading to power laws in SOC models (see SORNETTE 2006 Chapter 15; Chen and Wu 2010; Corral et al. 2008). In this section correlation between clusters were discussed.

\section{Two-Dimensional Random Domino Automaton with Constant $\mu$}

We investigate RDA with constant rebound parameter $\mu$ on a 2D square grid. Cells are called neighbours, if they have one side in common, but not if they only touch at one corner. The $2 \mathrm{D}$ case is much more complex than 1D RDA because $i$-clusters have perimeter lengths $t\left(i, r_{i}\right)$ depending on the cluster size $i$ and, moreover, on different geometric configurations $r_{i}$ of $i$-clusters. Because of these complexities, instead of deriving full equations for $n_{i}$ we are presenting only a draft of the balance equation. We use the mean perimeter $t(i)$ averaged over all possible configurations $r_{i}$.

A number $n_{i}$ of clusters of size $i$ can increase if a new point hits an empty cell on the perimeter of a $(i-1)$-cluster and the cell becomes occupied. The probability is $C v t(i-1) n_{i}-1$. The parameter $C$ denotes the probability that the empty cell does not belong to a perimeter of any other cluster (to avoid the linking process). On the other hand, if the empty cell belongs to the overlap of perimeters of two, three or four clusters, whose common size is $i-1$, then these clusters join in a cluster of size $i$. Estimation of the probability of this process is too complex for $2 \mathrm{D}$ RDA, so we denote it as the "fusion term".

Losses of $n_{i}$ can be due to hitting an empty cell (and changing its state) on a perimeter of $i$-cluster. The probability is $v t(i) n_{i}$ or if the point hits an occupied cell of the $i$-cluster and triggers the avalanche then the probability is $\mu i_{i}$. Therefore, the draft of the balance equation has the form:

$$
\mu i n_{i}+v t(i) n_{i}=C v t(i-1) n_{i-1}+v \cdot(\text { fusion term }) .
$$




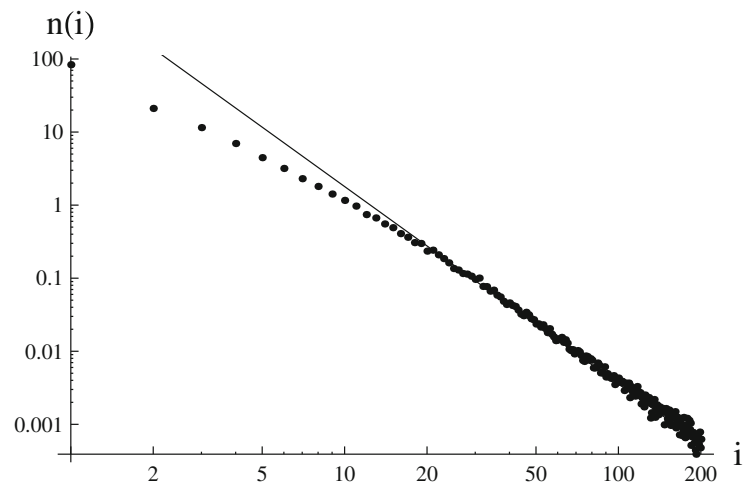

Figure 6

Cluster size distribution $n_{i}$ generated by 2D RDA with constant $\mu(i)=\delta$ and $\theta=1$ (points). Line presents the inverse power function with $\alpha=-2.7$

From the percolation theory (STAUFFER and Aharony 1992) we use the relation $t(i) \sim i$, valid for large clusters. If we put $t(i)=a i+b$ to Eq. (10) then the equation for the number of $i$-clusters in stationary state is:

$n_{i}=\frac{1}{i(a+\mu / v)+b}\left[(a i+b-a) C n_{i-1}+(\right.$ fusion term $\left.)\right]$.

\section{Correspondence Between Privilege Mechanisms in $1 D$ RDA and in $2 D$ RDA with Constant $\mu$}

The comparison of the draft Eq. (11) with the respective equation for 1D RDA with $\mu(i)=\delta / i^{2}$ [i.e., the third of the Eq. (1) set, in which $c=1-n_{1}^{0}$ / $n, d=n_{1}^{0} / n^{2}$ and numerator and denominator were multiplied by $i]$ :

$$
n_{i}=\frac{1}{2 i+\delta / v}\left[2 i c n_{i-1}+i d \sum_{k=1}^{i-2} n_{k} n_{i-1-k}\right]
$$

shows a close resemblance of both equations. The "fusion term" in Eq. (11) contains sums of products of two, three and four cluster distributions and strongly depends on geometric configurations of these clusters, which are associated with their perimeters. Therefore, the factor $i$ should appear in the term (like in Eq. 12).

Let us check if the similar Eqs. (11) and (12) can lead to similar distributions for $n_{i}$. In the case of $2 \mathrm{D}$

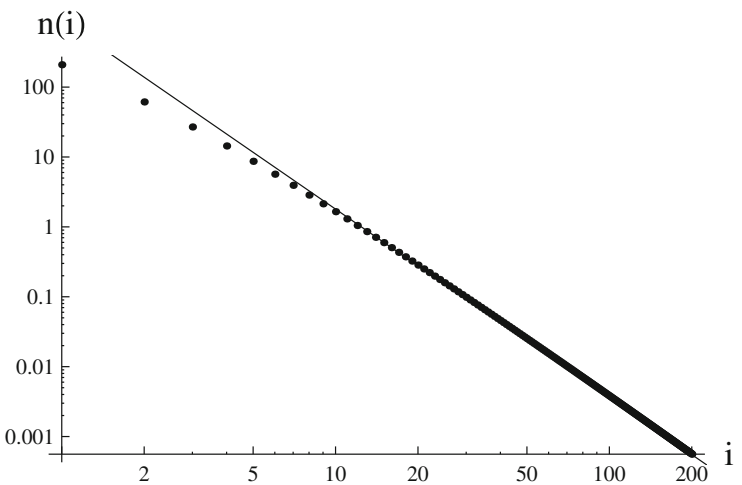

Figure 7

Cluster size distribution $n_{i}$ generated by 1D RDA with $\mu(i)=\delta / i^{2}$ and $\theta=7.74$ (points). Line presents the inverse power function with $\alpha=-2.7$

RDA we base it on numerical simulations. The distribution function of clusters calculated from the generated data is shown in Fig. 6. The distribution has an inverse power tail with the fitted exponent 2.7. For 1D RDA we can use Eq. (1). Then, assuming $\mu(i)=\delta / i^{2}$ and $\delta / v=7.74$, the distribution of $n_{i}$ has also the inverse power tail with exponent 2.7 (see Fig. 7).

Moreover, in the case of 1D RDA we can also apply another approach. The 1D automaton has an important feature in that it provides one-to-one correspondence between rebound parameters and size distribution of clusters (BIAŁECKI and CZECHOWSKI 2013). Therefore, we are going to reconstruct such a function $\mu(i)$, which can give the pure inverse power distribution $n_{i} \sim i^{-2.7}$ in the finite range $i=1, \ldots$, $K$ (for $i>K$ we put $n_{i}=0$ ). We use the following relations:

$$
\begin{aligned}
\frac{\mu(1)}{v}= & \frac{1}{n_{1}}\left[(1-\rho) N-2 n+n_{1}^{0}\right]-2 \\
\frac{\mu(2)}{v}= & \frac{n_{1}}{n_{2}}\left(1-\frac{n_{1}^{0}}{n}\right)-1 \\
\frac{\mu(i)}{v}= & \frac{1}{i}\left[2\left(\left(1-\frac{n_{1}^{0}}{n}\right) \frac{n_{i-1}}{n_{i}}-1\right)+\frac{n_{1}^{0}}{n_{i}} \sum_{k=1}^{i-2} \frac{n_{k} n_{i-1-k}}{n^{2}}\right] \\
& i \geq 3 .
\end{aligned}
$$

In these equations the parameter $n_{1}^{0}$ is found numerically from the implicit equation 


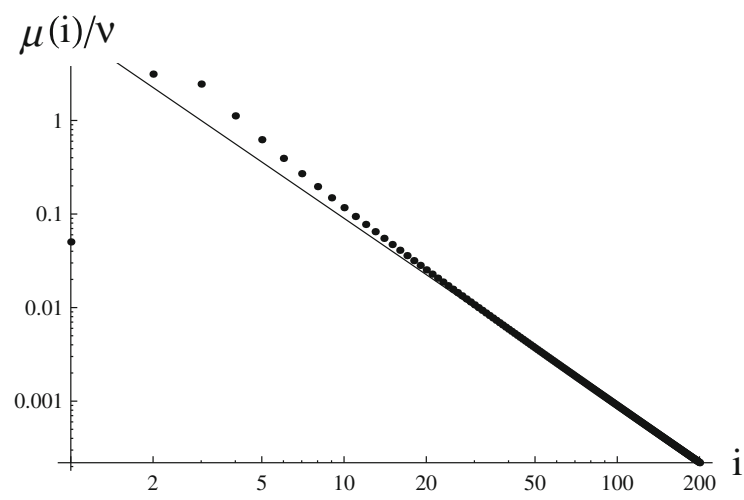

Figure 8

The rebound function $\mu(i) / v$ (points) reconstructed from Eq. (13), where $n_{i}=650 / i^{2.7}$ for $i=1, \ldots, 200, \rho \approx 0.3281$. Line presents the inverse-power function with $\alpha=-2$

$$
n_{1}^{0}=\frac{2 n}{3+\frac{2}{v n} \sum_{i \geq 1} i \mu\left(i, n_{1}^{0}\right) n_{i}}
$$

in which for $\mu(i)$ we put Eqs. (13) and the summation is over the finite range $1,2, \ldots, K$. The reconstructed $\mu(i)$ is shown in Fig. 8. For $i>20$ the function has an inverse power shape with the exponent close to two (continuous line).

The origin of long tails in this 1D RDA has been discussed in Sect. 3.1. This is a mechanism of privilege of greater clusters related to the survival rate given by the assumed rebound function $\mu(i) \sim 1 / i^{2}$. In the case of 2D RDA with constant $\mu$, the privilege has a geometric nature. The greater clusters have longer perimeters $t(i)$, which means greater probability of growth of these clusters due to linking of neighbouring clusters, when a site on the intersection of their perimeters becomes occupied. As a result, the number of greater clusters increases and long tail cluster size distribution appears. The mechanism was analyzed in (Czechowski 2003) for the case of the percolation process on Bethe and the 2D lattice.

In 1D RDA we have a constant perimeter equal to two for every cluster, but the survival rate (which increases with cluster size) can fulfill the role of the geometric privilege. In this meaning the simple 1D RDA mimics and explains behavior of the more complex 2D model.

In seismology both our automata reflect two main properties of earthquake processes: slow accumulation of energy at a constant rate to the fault, caused by the relative movement of tectonic plates, and an abrupt release of energy in earthquakes (avalanches). The motivations for using such simple automata are as follows: full physical modeling of earthquake process is an extremely complex task; therefore, many details must be averaged or simplified in some way. Moreover, the rules of the automata can be very simple, but they can lead to complex evolution of the system and produce rich patterns. The description of models by handy mathematical equations enables us to understand relations between microscopic mechanisms [e.g., given by the rebound function $\mu(i)$ ] and macroscopic characteristics of seismic phenomena, as, for example, the energy frequency power distribution or recurrence interval statistics (BIAŁECKI 2012b).

\section{Conclusions}

The tasks put forward in the introduction could be achieved only because we had at our disposal 1D RDA introduced earlier. This cellular automaton has simple rules, but this feature let us derive analytical equations describing the statistically stationary state. In particular, due to the analytical description, the influence of correlations on a form of cluster size distribution can be analyzed.

First of all, we have shown how the choice of rebound function $\mu(i)$ generates the form of $n_{i}$. The faster $\mu(i)$ is decreasing, the longer the tail of cluster size distribution. For constant $\mu(i)=\delta$, exponential tails are generated; however, for $\mu(i)=\delta / i^{2}$ the tail is described by a power law. We explain this behaviour by the privilege mechanism. The probability of survival of larger clusters is greater than that of smaller clusters (larger clusters are privileged).

The role of the parameter $\delta$ in the rebound function $\mu(i)$ was also analyzed. It describes the probability of triggering an avalanche irrespectively of the cluster size, if a point hits a randomly chosen occupied cell. Decreasing of the parameter causes a lengthening of tails; however, this regularity should be referred rather to the rise of density on the grid.

When the density is big enough, the role of correlations between clusters on the grid becomes essential and the analytical Eq. (1) is not valid. Comparison between analytical solutions and simulation results explains the role of correlations. Correlations induce a 
lengthening of the tail and a remarkable flattening of the distribution to a straight line. Even for constant $\mu(i)=\delta=0.002$ (then density $\rho \approx 0.81$ ), the cluster size distribution obtained from simulations resembles a power law in some range of $i$.

For small and moderate densities; however, the correlations are weak and can be omitted. Then the analytical description is valid. We have shown that equations for 1D RDA with $\mu(i)=\delta / i^{2}$ are similar to appropriate equations for 2D RDA with constant $\mu(i)=\delta$. In this way we can state that the simple 1D RDA mimics and explains the behavior of a more complex 2D model. The probability of survival of clusters [given by $1 / \mu(i)$ ] in $1 \mathrm{D}$ case refers to the probability of growth of clusters [given by the cluster perimeter $t(i)]$ in the $2 \mathrm{D}$ case, but both describe some privilege of larger clusters.

An inspiration to the concept of privilege, which can be treated as a generalization of Simon's (SIMON $1955,1960)$ idea of preferential attachment, was an analysis of the coagulation equation in the crack fusion problem (CzECHOwski 1993). In our papers (CZechowski 2001, 2003, 2005; CZEChowski and RoZMARYNOWSKA 2008), it was shown that in many cases (i.e., percolation processes, cellular automata, Cantor set, resource redistribution model, return-to-the-origin problem in random walk, multiplicative processes, and multiplication of probabilities) the hidden privilege can be extracted and explained. Moreover, a relevance between the privilege concept and the nonlinear approach was found. The present paper is a continuation of those investigations.

\section{Acknowledgments}

This work was partially financed by the project (Contract No. DEC-2012/05/B/ST10/00598) carried out by the Institute of Geophysics, Polish Academy of Sciences on the order of the National Science Centre.

Open Access This article is distributed under the terms of the Creative Commons Attribution License which permits any use, distribution, and reproduction in any medium, provided the original author(s) and the source are credited.

\section{REFERENCES}

Aschwanden, M., ed., Self-Organized Criticality Systems (Open Academic Press, Berlin Warsaw 2013).

BiaŁecki, M. (2012a), Motzkin numbers out of Random Domino Automaton, Physics Letters A 376, 3098-3100.

BIAŁECKI, M. (2012b), An explanation of the shape of the universal curve of the Scaling Law for the Earthquake Recurrence Time Distributions, arXiv:1210.7142 [physics.geo-ph].

Biąecki, M. (2013), From statistics of avalanches to microscopic dynamic parameters in a toy model of earthquakes, Acta Geophysica 61, no 6, 1677-1689.

BiAŁECKI, M., and CZECHOWsKi, Z. (2013), On one-to-one dependence of rebound parameters on statistics of clusters: exponential and inverse-power distribution out of Random Domino Automaton, J. Phys. Soc. Jpn. 82, 014003 (9 pp).

CHEN, H.S., and WU, G.Y. (2010), Effects of pair correlation on meanfield theory of BTW sand pile model, Physica A 389, 2339-2350.

Corral, A., Telesca L., and Lasaponara, R. (2008), Scaling and correlations in the dynamics of forest-fire occurrence, Phys. Rev. E 77, 016101, doi:10.1103/PhysRevE.77.016101.

Czechowski, Z., (1993), A kinetic model of nucleation, propagation and fusion of cracks, J. Phys. Earth 41, 127-137.

CZechowski, Z., (2001), Transformation of random distributions into power-like distributions due to non-linearities: application to geophysical phenomena, Geophys. J. Int. 144, 197-205.

CZEchowski, Z., (2003), The privilege as the cause of the power distributions in geophysics, Geophys. J. Int. 154, 754-766.

Czechowski, Z., (2005), The importance of the privilege in resource redistribution models for appearance of inverse-power solutions, Physica A 345, 92-106.

Czechowski, Z., and Rozmarynowska, A. (2008), The importance of the privilege for appearance of inverse-power solutions in Ito equations, Physica A 387, 5403-5416.

CZEChowski, Z., and BiAŁecki, M. (2012a), Ito equations out of domino cellular automaton with efficiency parameters, Acta Geophysica 60, no 3, 846-857.

CZECHOWski, Z., and BiAŁECKI, M. (2012b), Three-level description of the domino cellular automaton, Journal of Physics A: Math. Theor. 45, 155101 (19 pp).

Paczuski, M., and BAK, P. (1993), Theory of the one-dimensional forest-fire model, Phys. Rev. E 48, R3214-R3216.

Simon, H.A. (1955). On a class of skew distribution functions. Biometrika 42, 425-440.

Simon, H.A. (1960). Some further notes on a class of skew distribution functions. Information and Control 3, 80-88.

Sornette, D., Critical Phenomena in Natural Sciences (SpringerVerlag, Berlin Heidelberg 2006).

Stauffer, D., and Aharony, A., Introduction to Percolation Theory (Taylor and Francis, London 1992). 